\title{
Hippocampal neuroplasticity and inflammation: relevance for multiple sclerosis
}

\author{
Andrea Mancini ${ }^{1}$, Lorenzo Gaetani ${ }^{1}$, Maria Di Gregorio ${ }^{1}$, Alessandro Tozzi ${ }^{2,3}$, Veronica Ghiglieri ${ }^{2,4}$, \\ Paolo Calabresi ${ }^{1,2}$ and Massimiliano Di Filippo ${ }^{1 *}$
}

\begin{abstract}
Cognitive impairment is very frequent during multiple sclerosis (MS), involving approximately $40-70 \%$ of the patients, with a profound impact on patient's life. It is now established that among the various central nervous system (CNS) structures involved during the course of MS, the hippocampus is particularly sensitive to the detrimental effects of neuroinflammation. Different studies demonstrated hippocampal involvement during MS, in association with depression and cognitive impairment, such as verbal and visuo-spatial memory deficits, even during the earlier phases of the disease. These cognitive alterations could represent the visible consequences of a hidden synaptic impairment. Indeed, neuronal and immune functions are intertwined and the immune system is able to modulate the efficacy of synaptic transmission and the induction of the main forms of synaptic plasticity, such as long term potentiation (LTP). Hippocampal synaptic plasticity has been studied during the last decades as the physiological basis of human learning and memory and its disruption can be associated with behavioral and cognitive abnormalities. The aim of the present work is to review the available evidence about the presence of hippocampal synaptic plasticity alterations in experimental models of MS, specifically during the course of experimental autoimmune encephalomyelitis (EAE) and to discuss their relevance with regard to human MS. Indeed, the failure of synapses to express plasticity during neuroinflammation could potentially lead to a progressive failure of the brain plastic reserve, possibly contributing to disability progression and cognitive impairment during MS.
\end{abstract}

Keywords: Hippocampus, Multiple sclerosis, Synaptic plasticity, Neuroinflammation, Experimental autoimmune encephalomyelitis, Cognitive impairment

\section{Background}

Neurons and synapses are located behind the bloodbrain barrier and the anatomical and functional characteristics of cerebral perfusion led to the erroneous believing that the physiology of neuronal communication could not be influenced by immune responses. Conversely, it is now well established that neuronal function is strongly influenced by both central and peripheral inflammation [1]. In particular, it has been shown that the immune system is able to modulate

\footnotetext{
* Correspondence: massimiliano.difilippo@unipg.it

${ }^{1}$ Clinica Neurologica, Dipartimento di Medicina, Università degli Studi di Perugia, Ospedale Santa Maria della Misericordia, S. Andrea delle Fratte, Perugia 06132, Italy

Full list of author information is available at the end of the article
}

the efficacy of synaptic transmission and the induction of the main forms of synaptic plasticity [1]. Glial and microglial cells are now recognized as active elements of synapses, playing a central role in neuro-inflammatory processes [2-4]. Moreover, several soluble products that were thought to exclusively exert immunological functions, such as cytokines, are known to influence synaptic transmission during both physiological and pathological conditions $[1,5]$. In particular, the inflammatory demyelinating process taking place during multiple sclerosis (MS), as well as systemic inflammatory triggers, could lead to the anomalous production of inflammatory mediators at synaptic sites with the subsequent disruption of synaptic homeostasis. 
Systemic infections and chronic inflammatory processes are associated with behavioral and cognitive changes consisting in fatigue, depression, impaired learning and memory. These symptoms, all very frequent during MS, were collectively named as "sickness behavior" [6-8]. Specifically, during MS, cognitive impairment is very common with an important impact on patient's daily activities and quality of life [9] and it is detectable even during the earlier phases of the disease $[9,10]$. Behavioral and cognitive alterations during MS could represent the clinical consequences of a subtle synaptic impairment, derived from an abnormal production of pro-inflammatory molecules by activated microglia during central inflammation $[1,11,12]$. Altered synaptic plasticity could shape the course of MS. Indeed, the plastic potential of the central nervous system (CNS) is fundamental to recover from brain injuries, and the disruption of the main forms of synaptic plasticity may exert a negative impact on disease progression and accumulation of disability $[13,14]$. Among the various CNS sites involved in MS, the hippocampus is particularly vulnerable to the detrimental effects of neuroinflammation $[15,16]$. Several studies demonstrated that hippocampal impairment is associated with depression and cognitive decline, such as verbal and visuo-spatial memory deficits [17-20]. The aim of the present work is to review the available evidence about the alteration of hippocampal synaptic plasticity in experimental models of MS and to discuss their relevance with regard to human MS.

\section{The hippocampus: structure and function}

"The findings reported herein led us to attribute a special importance to the anterior hippocampus and hippocampal gyrus in the retention of new experience." (Scoville and Milner, 1957) [21].

This sentence was the conclusion reached by Scoville and Milner during their neurosurgical study in 1957 [21]. In order to treat patients with psychiatric disorders or untreatable forms of epilepsy, they performed extensive bilateral medial temporal-lobe resections. One of their patient, H.M., after a bilateral medial temporal lobe resection, showed a partial retrograde and anterograde amnesia [21]. Interestingly, his perceptual abilities were spared, together with his working memory and motor skill learning. This observation contributed to a large research field about memory and its anatomical and biological background. During the last decades, many neuropsychological, behavioral and neuroimaging studies pointed to the medial temporal lobe, in particular to the hippocampus, as the critical cerebral structure for declarative and spatial memory [22, 23]. In particular, the integrity of human hippocampus is considered fundamental for the formation of episodic memory, the ability to recall personal experiences and semantic knowledge, that could be considered the basis of personal learning and culture [22, 23]. This may explain why an impairment of these cognitive functions appears early together with a bilateral atrophy of medial temporal lobes during Alzheimer's disease [22] and why during transient global amnesia a bilateral hippocampal dysfunction occurs [24]. Moreover, the hippocampus seems to be the core structure encoding visuo-spatial information, as explained by the cognitive map theory proposed by O'Keefe and Nadel $[25,26]$. This theory tries to explore the functional organization of hippocampus in rats and other animals, describing a cognitive map located in this cerebral structure, with neurons acting as place cells [25]. Place cells represent the functional units of this map, selectively increasing their firing rate when animals explore a determined and unique spatial region, helping the rodent to orientate itself and leading to the formation of an allocentric spatial memory [25, 27]. The importance of the hippocampus in spatial learning is also confirmed by the evidence that hippocampal volume is linked to spatial ability in different species of birds and small mammals $[28,29]$. In humans, neuroimaging studies with structural magnetic resonance imaging (MRI) scans showed that the posterior hippocampal volume was increased bilaterally in licensed London taxi drivers, compared to age-matched controls [30]. This increased volume can be explained by the extensive training in spatial navigation made by these workers, as it correlates positively with time spent driving through the chaotic traffic in the British capital [30]. In conclusion, the hippocampus appears to be fundamental to answer questions about what, when and where something takes place and hippocampal involvement has been correlated to deficits in verbal and visuo-spatial memory during MS [17, 20, 31].

The hippocampal structure and synaptic plasticity were studied to assess their relationship with memory and human behavior. Declarative and episodic memory can be deconstructed in sequentially organized associative representations, in which each single event or variable is specifically settled in a unique flow, ultimately leading to a coherent experience or meaning [23]. Memory could be seen as a relational network in which each event correlates with others in a negative or positive way. Hippocampal structure and synaptic properties seem to fit perfectly with this functional activity, owing to its ability to create associations, intensifying some of them while lowering others [27, 32, 33]. This structure is well organized to process and associate different kinds of information, working as a C-shaped computational loop with extensive recurrent fibers (Fig. 1). A lot of 


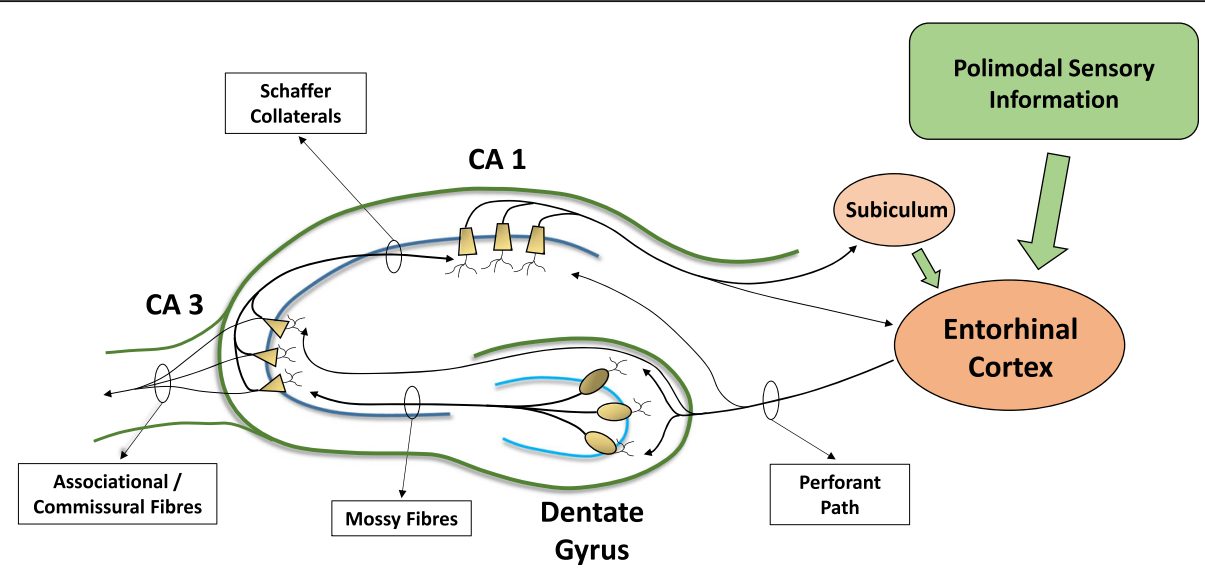

Fig. 1 Simplified functional anatomy of the hippocampus. Hippocampal circuitry is usually described as a trisynaptic loop, with a C-shaped organization. Perforant Path (PP) carries the major hippocampal input, consisting in polimodal sensory information collected by the Entorhinal Cortex (EC) from higher sensory cortices. PP contacts dendritic spines of granule cells in hippocampal Dentate Gyrus (DG), and a smaller part contacts directly neurons of the CA1 and CA3 hippocampal areas. Axons from DG granule cells are collectively named Mossy Fibres (MF) and project to hippocampal CA3 pyramidal cells. These cells represent an important computational node, since they are connected to contralateral CA3 and CA1 pyramidal cells through associational/commissural fibres. Moreover, they receive recurrent connections from ipsilateral CA3 cells, representing "internal" inputs, and they receive "external" inputs from DG and EC. CA3 pyramidal cells then project to ipsilateral CA1 pyramidal neurons, with connection fibres named Schaffer Collaterals (SC). Synapses between SC and CA1 neurons, and their plastic modulation, seem to be crucial for hippocampal computational ability and memory encoding. Lastly, CA1 axons contact directly and indirectly, through Subiculum, the EC

information from virtually all cortical associative areas converge towards the hippocampus through entorhinal cortex (EC) and perforant path (PP). PP axons make excitatory synaptic contact with all the three major areas of the hippocampus, the dentate gyrus (DG), cornus ammonis (CA) 3 and CA1 areas. In particular, they contact granule cells located within the DG. These cells project through the mossy fibres (MF) to CA3 pyramidal neurons, which in turn send recurrent connections to other CA3 pyramidal neurons creating a kind of reverberant signaling. Thus, CA3 hippocampal area receives two kinds of information: "external" information from EC, PP and CA1 area through the MF; "internal" information from CA3 pyramidal recurrent connections [34]. Axons from CA3 neurons finally converge into Schaffer Collaterals (SC) and contact dendrites of CA1 pyramidal cells, whose projections ultimately contact the subiculum (SUB) and the EC [23, 33, 34]. This circuitry is extensively modulated by associational and commissural fibers and by inhibitory neurons distributed in all of the described hippocampal areas, creating a complex net of connections.

The plastic modulation of hippocampal synaptic activity could represent the functional basis of its computational ability. The simultaneous and repetitive stimulation of connected sub-components of this assembly, each one representing a specific external or internal stimulus, could lead to a strengthened connection between them [23, 33, 34]. Cortico-hippocampal connections act as an individual guidance to modulate behavior in relation to the spatial, temporal and semantic context [27]. Such computational activity could predict upcoming stimuli and events in the environment, expanding its role from a static representation of reality to a probabilistic computational unit, useful to orientate human behavior towards a mutating and unexpected reality [27].

\section{Synaptic plasticity, memory and learning in the hippocampus}

\begin{abstract}
"Our experiments show that there exists at least one group of synapses in the hippocampus whose efficiency is influenced by activity which may have occurred several hours previously - a time scale long enough to be potentially useful for information storage." (Bliss and Lomo, 1973) [35]
\end{abstract}

The way through which a neuronal network can storage and recall information always attracted neuroscientist's attention. Since the histological structure of the nervous system was described, several researchers tried to hypothesize how neural activity can modulate and influence neuronal connections [36]. In 1949, Donald Hebb in his book "The organization of behavior" postulated that a connection between two neuronal cells is strengthened by the simultaneously activation of both of them [37]. This Hebbian principle is a coincidencedetection rule, which reflected the long-standing idea, dating back to Cajal, that repeated stimulation of a synaptic connection could induce structural synaptic 
changes, as a way to form sustained memory traces from episodic experiences during learning [38]. Few decades later, an experimental support to this principle was provided. Bliss and Lomo demonstrated that repetitive high frequency stimulation (HFS) of the PP fibers to the DG of the hippocampus leads to a persistent average increase in the amplitude of the population excitatory post-synaptic potential (EPSP), meaning a powerful response of hippocampal granule cells [35]. This effect was firstly named long-lasting potentiation, successively best known as long-term potentiation (LTP). To date, hippocampal LTP is surely the best known activitydependent form of synaptic plasticity [39], it is expressed by excitatory synapses throughout the brain and it appears to be dependent both on post-synaptic and pre-synaptic processes [40] (Fig. 2). LTP, together with long-term depression (LTD) [40,41] and other forms of synaptic plasticity, represent the most probable physiological basis of human learning and memory [23, 27, 33, 34], and several studies showed their occurrence in vivo at synaptic sites $[40,42]$.

Long-term forms of synaptic plasticity, such as LTP, are considered the physiological basis of human memory, since they are induced rapidly after synaptic stimulation, they are stable over time and can associate different stimuli in a functional network facilitating the encoding and recalling of information [42]. To prove these features of LTP many studies tried to demonstrate with electrophysiological techniques that it plays an important functional role in learning and memory. In 1983, Collingridge and colleagues showed that the selective blockage of $N$-methyl-D-aspartate (NMDA) glutamate receptors with the drug amino-phosphonovaleric acid (AP5) at excitatory hippocampal synapses, was able to

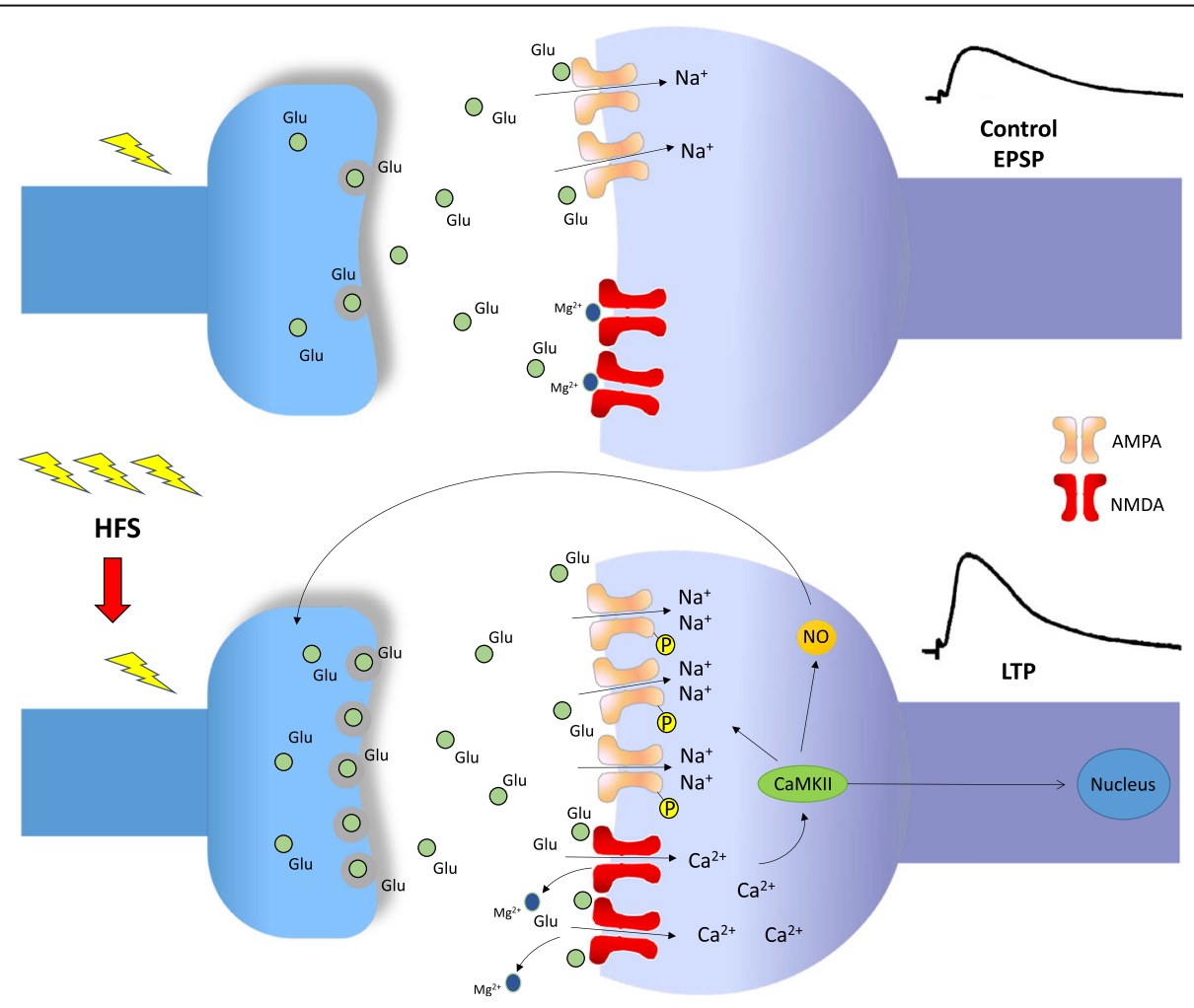

Fig. 2 Long-term potentiation (LTP) induction. In basal "control" condition, glutamate (Glu) released by the pre-synaptic terminal after an electric stimulation interacts with a-amino-3-hydroxy-5-methyl-4-isoxazolepropionic acid (AMPA) glutamate receptor, resulting in sodium ( $\mathrm{Na}^{+}$) influx and an excitatory post-synaptic potential (control EPSP). In this condition, glutamate interaction with N-methyl-D-aspartate (NMDA) glutamate receptors has no consequence because of the voltage-dependent blockage by magnesium $\left(\mathrm{Mg}^{2+}\right)$ of these receptors. During repetitive synaptic stimulation, which is mimicked in experimental conditions by the high frequency stimulation (HFS) protocol, the voltage-dependent NMDA blockage by Mg ${ }^{2+}$ is removed, allowing a $\mathrm{Ca}^{2+}$ influx into the post-synaptic element. This influx leads to the activation of calcium/calmodulin-dependent protein kinase II (CaMKII) and other several kinases, not shown in the figure, such as protein kinase A (PKA), atypical protein kinase C isoforms and mitogen-activated protein kinases (MAPK), which induce molecular changes in the post-synaptic dendritic spine [1, 40]. AMPA receptors are phosphorylated with an increase in $\mathrm{Na}^{+}$ conductance, and more AMPA receptors are delivered to the plasma membrane from the sub-synaptic compartments. Moreover, neuronal gene expression is modulated in order to modify the morphology and the molecular structure of the dendritic spine. Finally, retrograde messengers like nitric oxide (NO), seem to play a role in LTP induction and maintenance, enhancing Glu release from the pre-synaptic element. All these synaptic modifications ultimately result in a sustained long-term increase of the EPSP (LTP), enhancing excitatory transmission between the two neurons 
block the induction of LTP [43]. The role of NMDA glutamate receptor in synaptic plasticity was later supported by the studies of Morris and colleagues, who studied the behavioral effects of chronic, in vivo, intraventricular infusion of D,L-AP5 in rats [44]. During their experiments, rats were located into a large pool of water with a hidden platform located at a corner, and they measured the mean time needed to find the hidden platform and escape from water, a test known as Morris water maze [44]. The authors showed that the infusion of a selective NMDA antagonist, capable to block LTP induction in vitro, caused a significant impairment in learning and spatial navigation, suggesting a key role for hippocampal LTP in memory and spatial learning [44]. Whitlock and colleagues studied hippocampal synaptic alterations induced by inhibitory avoidance test (IAT), a test capable to induce a rapidly acquired and stable spatial memory, with associated changes in gene expression in the CA1 area of hippocampus [42]. They showed that the molecular changes occurring at hippocampal synapses during the in vitro induction of LTP, like the phosphorylation of $\alpha$-amino-3hydroxy-5methyl-4-isoxazolepropionic acid (AMPA) glutamate receptor GluR1 subunit, where similar to those occurred after a cycle of active avoidance training, meaning that synapses responsible of controlling that form of learning probably expressed LTP [42]. These results support the hypothesis that spatial learning induces LTP in the CA1 area of the hippocampus.

In the last years, many studies explored new physiological pathways and cellular types implicated in the modulation of activity-dependent synaptic plasticity. Resident glial cells in the CNS, like astrocytes, seem to be key partners of neurons, actively regulating synaptic transmission [45]. Interestingly, also microglial cells, the resident immune cells and phagocytes of CNS, which rapidly activate in response to brain injury, dynamically interact with synapses in physiological conditions, acting like synaptic sensors modulating neuronal excitability and transmission $[3,46]$. To date, synapses appear to be a more complex anatomical structure than it was believed before. Synapses are indeed now considered a "tri-partite" or even a "quad-partite" connection, in which pre- and post- synaptic neurons interact with glial cells such as astrocytes or microglia in order to modulate the efficacy of synaptic transmission [2, 3, 45-48]. Interestingly, the functional role of glial cells in synaptic plasticity could be crucial in particular during pathological conditions. For example, the regulation of water balance in CNS has been reported to be relevant in synaptic plasticity [49]. Aquaporin-4 (AQP4) is a water channel expressed by astrocytes in CNS and its abnormal modulation during pathologic conditions such as cerebral edema, epilepsy and ischemia could lead to a defective LTP and LTD at hippocampal synapses [50]. Increasing attention is now oriented towards the functional and structural role of astrocytes in synaptogenesis and the regulation of synaptic activity [5, 51, 52]. Astrocytes are able to modulate the extracellular concentration of neurotransmitters, but they can also influence the expression and functional activity of postsynaptic receptors [53], helping in the maintenance of advantageous connections and removal of superfluous ones. Moreover, neuronal homeostasis, energy metabolism and defense against oxidative stress appear to be dependent on several astrocytic processes such as glutamate uptake and recycling, $\mathrm{K}^{+}$buffering, lactate release, glycogen mobilization, glutathione (GSH) synthesis and the production of neuroprotective trophic factors (for example nerve growth factor, NGF, ciliary neurotrophic factor, CNTF, glial cell-line derived neurotrophic factor, GDNF, and fibroblast growth factor 2, FGF-2) [54]. During neuroinflammatory processes, glial activation could lead to the release of a wide repertoire of immune mediators and cytokines, potentially affecting neuronal viability and synaptic transmission [54]. Indeed, an astrocyte-derived mediator, such as lactosylceramide, could play a role in CNS inflammation and neuronal degeneration in experimental models of MS, interfering with glutamate transmission [55-57]. Similarly, microglia is gaining attention as a central regulator of synaptic transmission during neuroinflammation and other neurodegenerative disorders $[2,3,48,58]$.

\section{Microglia, neuroinflammation and synaptic plasticity: a deep impact}

In the CNS, approximately $10 \%$ of the total cellular elements are represented by microglia, with variations between 0.5 to $16.6 \%$ for each human brain area $[3,59]$. Microglia is constituted by mesodermal cells which migrate in the neural tube from the yolk-sac during the first ten embryonic days $[2,48]$. They share the same lineage with monocytes and macrophages and represent the main resident immune innate defense within the CNS, contributing to inflammatory response against different kind of injuries [3]. Microglia is normally resting in the mature nervous system, with a phenotype characterized by long ramified processes extending from a small cell body [2]. During nervous tissue injury or inflammatory processes, microglial cells become activated, shaping into a globular "amoeboid" form with the ability to move towards the site of damage [3]. Even when in their "quiescent" or "resting" state, the thin microglial processes are highly dynamic and continuously scan neighboring neurons transiently contacting synapses near them with fast movements [60, 61]. Interestingly, microglial thin processes express several classes of neurotransmitter receptors and ion channels, 
normally found in neurons [3]. It has been demonstrated that NMDA receptors expressed by microglia can modulate the neuroinflammatory process, influencing reactive oxygen species (ROS) and cytokines production [62]. This rich equipment in neurotransmitter receptors and ion channels could be useful in physiological conditions to sample the extracellular matrix, monitoring neuronal activity near microglial cells. This function could lead microglia to play the role of active supervisor of neuronal firing activity and synaptic function [2, 48]. The monitoring activity of microglia seems to be crucial in the CNS development and refinement during the postnatal period [47, 63]. Microglia is indeed able to phagocytize inappropriate exuberant synapses, a process known as synaptic pruning, and apoptotic neurons, even by directly inducing programmed cell death through apoptotic signals $[2,47,48,63]$. In fact, mice lacking the receptor for fractalkine (CX3CR1), a molecule which promotes microglia migration into CNS, show a reduced microglia brain concentration during CNS development, in association to an impaired synaptic pruning and synaptic transmission [63]. Parkhurst and colleagues studied the role of microglia in synaptic plasticity in an animal model lacking CNS microglia [64]. They generated CX3CR1(CreER) mice expressing tamoxifen-inducible Cre recombinase that allows for specific gene function manipulation in microglia [64]. They induced the expression of diphtheria toxin receptor in microglia, so they could later specifically remove microglia from the brain upon diphtheria toxin administration [64]. They found that microglia-depleted mice show behavioral abnormalities associated with hippocampal-dependent learning impairment. Interestingly, these abnormalities were associated with abnormal turnover of dendritic spines, and could be mimicked by the lack of brainderived neurotrophic factor (BDNF) [64]. These results obtained by Parkhurst strongly support the crucial role played by microglia in neuroplasticity and learning. Thus, emerging evidence is converging toward a regulating role for microglia on synaptic transmission and synaptic plasticity in the adult brain. Microglia can act as modulator by several mechanisms, for example producing molecules such as interleukin-1 $\beta$ (IL-1 $\beta$ ) and tumor necrosis factor $\alpha$ (TNF- $\alpha$ ), which have an active influence on the main forms of synaptic plasticity, learning and memory, both during physiological and pathological condition $[1,3,65,66]$.

In particular, the effect of IL-1 $\beta$ on synaptic plasticity was studied by Katsuki and colleagues, who showed that recombinant human IL-1 $\beta$ is able to block the induction of hippocampal LTP in mouse brain slices [67]. Other research groups confirmed this evidence, suggesting a key role for this cytokine in synaptic plasticity defects observed during neuroinflammatory processes $[58,66,68-70]$. However, endogenous IL-1 $\beta$ is normally expressed at low levels in control conditions, and it has been proposed to be essential for the physiological induction of hippocampal LTP and consolidation of memory [65, 71]. Accordingly, the analysis of a genetic murine model lacking IL-1 $\beta$ receptor showed impaired hippocampal neuro-plasticity [72]. The molecular pathway activated by IL-1 $\beta$, which involves mitogen activated protein kinases (MAPKs) and nuclear factor-kappa $\beta$ (NF-k $\beta$ ), could indeed influence neuronal gene expression and regulate plastic processes at synaptic sites [1]. In particular, the assembly, expression and phosphorylation of glutamate receptor, glutamate secretion, intracellular calcium concentration, ROS production and cholinergic neurotransmission are influenced and regulated by IL- $1 \beta$ [1]. Thus, IL- $1 \beta$ could exert a fundamental role in modulating and favoring synaptic plasticity when expressed at physiological low levels, while an increased expression of this cytokine, such as during neuroinflammatory processes, could exert detrimental effects on neuroplasticity in the hippocampus. Interestingly, a recent study assessed the cerebro-spinal fluid (CSF) levels of this cytokine in 170 patients with relapsing-remitting MS, during clinical and radiological remission [73]. As result, higher CSF levels of IL-1 $\beta$ were found to be associated with midterm disease progression [73]. This work could support the hypothesis that an inflammatory environment in the CNS, potentially leading to the failure of brain plastic capacities, could play a pathogenic role during disease progression in patients with MS.

The same observation seems to be true for microglial cells. Indeed, although their physiological activity is necessary for neuronal circuit development and function, when microglia becomes activated by inflammatory stimuli it is able to disrupt the ability of CNS synapses to express plasticity. In particular, inflammatory processes, like those occurring during EAE and MS, alter the fine balance existing between microglia and synapses/neurons, leading to excessive secretion of inflammatory products, such as cytokines and ROS, which could be harmful for neurons and neuroplastic processes $[1,3,74]$.

\section{Hippocampal synaptic plasticity in experimental models of MS}

The possibility to mimic the complex pathogenic mechanism occurring during human MS still remains very difficult. The experimental model most frequently utilized to study human MS is represented by experimental autoimmune encephalomyelitis (EAE), since it mimics some of the clinical, immunological and histopathological features of MS [75]. This model resembles the pathogenesis of MS, 
since it is characterized by the induction of a myelinspecific immune response trough subcutaneous injections, for example, of myelin oligodendrocyte glycoprotein 35-55 $\left(\mathrm{MOG}_{35-55}\right)$ or a syngeneic spinal cord homogenate $[76,77]$. The characterization of this model led to important discoveries about the pathogenesis of inflammatory demyelination. The study of hippocampal synaptic plasticity dysfunction occurring during EAE has been conducted by few laboratories, but it represents an important acquisition to understand the course of the disease [13]. Kim Do and colleagues studied the alteration of hippocampal synaptic plasticity during EAE induced in $\mathrm{C} 57 / \mathrm{Bl6}$ mice through immunization against $\mathrm{MOG}_{35-55}$ [78]. They showed a synaptic dysfunction in the hippocampal CA1 area, with a selective impairment in the ability to express LTP at two different time points post-immunization (13-16 days and 25-35 days), corresponding approximately to two disability stages (peak and milder). Interestingly, even before motor deficits became evident, mice manifested deficits in spatial learning [78], an evidence also confirmed by other groups [79] consistent with the hypothesis of a latent hippocampal dysfunction during the earlier phases of the disease, just after the beginning of the inflammatory process induced by the administration of the myelin-specific immunological trigger. As an attempt to reverse the observed synaptic deficit, authors fed EAE mice with a ketogenic diet [78]. Interestingly, the ketogenic diet was able to ameliorate the LTP impairment during EAE, in addition to the reduction of $\mathrm{CD}^{+}$cells and microglia/macrophages concentration in the CNS during active neuroinflammation, with subsequent reduced production of several pro-inflammatory cytokines (IL-1 $\beta$, interleukin- 6 , TNF- $\alpha$, interleukin-12, interleukin-17) and ROS [78]. These results support the hypothesis that some of the soluble products of inflammation are responsible for the observed hippocampal synaptic plasticity deficit during the earlier phases of the disease. More recently, another research group demonstrated a hippocampal LTP impairment in rats affected by EAE, with in vivo electrophysiological recordings [80]. In particular, during the acute phase of EAE (28 hours after mice obtained a disability score of at least 3 ) the authors described a suppressed LTP induction, probably related to an increased inhibitory GABA-mediated effect [80].

In our laboratory, we found an impaired LTP induction in the hippocampal CA1 area both during the acute inflammatory peak phase of EAE [68] and the later remission phase [58]. As experimental model for MS, we induced EAE in Biozzi $\mathrm{ABH}$ mice, which are particularly prone to develop autoimmune responses, by the injection of syngeneic spinal cord homogenate [68]. This model follows a clinical course, reminiscent of relapsingremitting MS, with acute phases characterized by detectable neurological signs followed by remissions with partial or complete recovery, with a later progressive phase [76]. During the first acute relapse of EAE, the LTP impairment was found to be associated with an intense activation of hippocampal microglia and an increased hippocampal concentration of IL-1 $\beta$ [68]. Such hippocampal synaptic plasticity disruption was associated with changes in the subunit composition of NMDA glutamate receptor in the hippocampus, with a reduced expression of GluN2B-subunit [68]. Several evidences suggested that GluN2B-subunit containing receptors are more prone to favor the induction of LTP in comparison to GluN2A containing ones [81, 82]. These molecular changes could be related to the known effects of IL-1 $\beta$ on gene expression, glutamate signaling and synaptic function [1], ultimately leading to a disruption of hippocampal LTP. Interestingly, synaptic impairment in the CA1 area of the hippocampus was detectable not only during acute EAE phase, but even in the remission phase of the experimental disease. We found that after the resolution of motor deficits, it was still possible to detect a significant impairment of hippocampal LTP and behavioural abnormalities, suggesting a deficit in spatial memory during the open field hole-board test [58]. These abnormalities in remitting EAE mice were associated with a persistent activation of hippocampal CA1 microglia, an increase in hippocampal IL-1 $\beta$ levels and the over expression by microglial cells of a ROS producing enzyme, nicotinamide adenine dinucleotide phosphate (NADPH) oxidase, as assessed by hippocampal immunohistochemistry and Western blot analyses [58]. The treatment with minocycline, an antibiotic with profound anti-inflammatory properties, was able to significantly reduce hippocampal microglia activation, NADPH oxidase expression and IL-1 $\beta$ levels, and to reverse the previously observed hippocampal LTP impairment and cognitive deficits in remitting EAE mice [58]. As a further support to the role of activated microglia and ROS producing enzymes, we showed that the application of apocynin, an inhibitor of NADPH oxidase, was able to rescue hippocampal LTP deficit during the EAE remission phase [58]. Moreover, apocynin was also able to block the detrimental effect of IL-1 $\beta$ on synaptic plasticity in control conditions, suggesting an involvement of NADPH oxidase expression in this process [58]. Thus, even after the resolution of motor symptoms in remitting EAE mice, persistent hippocampal microglia activation is associated with the release of inflammatory mediators, such as IL-1 $\beta$, and the production of ROS through NADPH oxidase, and could be responsible of hippocampal CA1 LTP impairment and related cognitive deficits in spatial learning during MS [58]. 
Recently, Novkovic and colleagues showed that in the early mild phase of $\mathrm{MOG}_{35-55}$ induced EAE (between days 14 and 19 post immunization), there was no impairment of hippocampal synaptic plasticity, while in later phases (between days 40 and 45 post-immunization) hippocampal LTP and LTP-related memory, such as spatial memory, were affected [83]. In their experimental setting they used as control group mice treated with complete Freund's adjuvant (CFA) alone, without $\mathrm{MOG}_{35-55}$ [83].

In contrast with the results described above, Nisticò and colleagues found an enhanced hippocampal LTP amplitude in slices obtained by EAE mice, with respect to CFA-treated mice, leading to the hypothesis that the neuro-inflammatory process associated with EAE could also potentiate neuroplastic abilities of the CNS [84] in the utilized experimental setting. This research group utilized $\mathrm{MOG}_{35-55}$ induced EAE, and they considered as control group mice treated with an emulsion of CFA. With this experimental protocol, they also showed that the ability of EAE in favouring LTP induction was mimicked by the exposure of control CFA slices to IL-1 $\beta$ [84]. Interestingly, during EAE they found a reduced efficacy of inhibitory GABAergic transmission, coupled with a reduction of hippocampal GABAergic neurons [84]. This effect was mimicked in CFA treated mice slices by the exposure to IL- $1 \beta$ or IL- $1 \beta$-activated microglial cells [84]. Considering this evidence, the authors suggested that the pathogenic mechanism underlying the enhanced synaptic plasticity during acute phase of EAE was represented by a decreased hippocampal inhibitory neurotransmission [84]. Finally, another research group described a normal hippocampal LTP during the course of EAE. Indeed, Prochnow and colleagues demonstrated that an LTD impairment could be detected in several brain regions during the acute phase of $\mathrm{MOG}_{35-55}$ induced EAE, such as the cerebellum and the superior culliculus [85]. Conversely, they did not find abnormalities of synaptic plasticity in the CA1 area of the hippocampus, with a normal expression of LTP and LTD [85].

In conclusion, hippocampal long-term synaptic plasticity during experimental MS has been studied by several research groups with partially contrasting results [13]. The majority of the studies found a reduction of the synapse's ability to express LTP, but reports also describe a normal and even an enhanced LTP during the course of EAE. Unfortunately, the existing differences in experimental models (animal used, modality of EAE induction, CFA treated or untreated mice as control group) and electrophysiological protocols (time points after EAE induction, specific electrophysiological technique, stimulation protocols, clinical severity of EAE symptoms) interfere with the interpretation and comparison of the studies [13]. However, it appears clear that during CNS inflammation there is significant alteration of the neuronal ability to express the main forms of synaptic plasticity, probably due to the effect of activated microglia and soluble products of inflammation (Fig. 3). These abnormalities in synaptic plasticity could be a dynamic process, influenced by the severity of inflammation and the stage of the disease, underlying a progressive functional failure of the brain plastic reserve, possibly contributing to disability progression during human disease. In line with this hypothesis, the ability to express synaptic plasticity has been proposed as a critical factor counteracting disability progression in MS [86]. Mori and colleagues showed that an altered brain ability to express LTP is associated with incomplete symptom recovery after an acute relapse and accumulation of disability [87]. Interestingly, the same group also demonstrated that the ability to express LTP, explored by transcranial magnetic stimulation (TMS) over the primary motor cortex, was still possible in stable relapsing-remitting MS patients, while it was absent in primary progressive MS suggesting that the brain plastic reserve might be crucial to contrast clinical deterioration in MS [88].

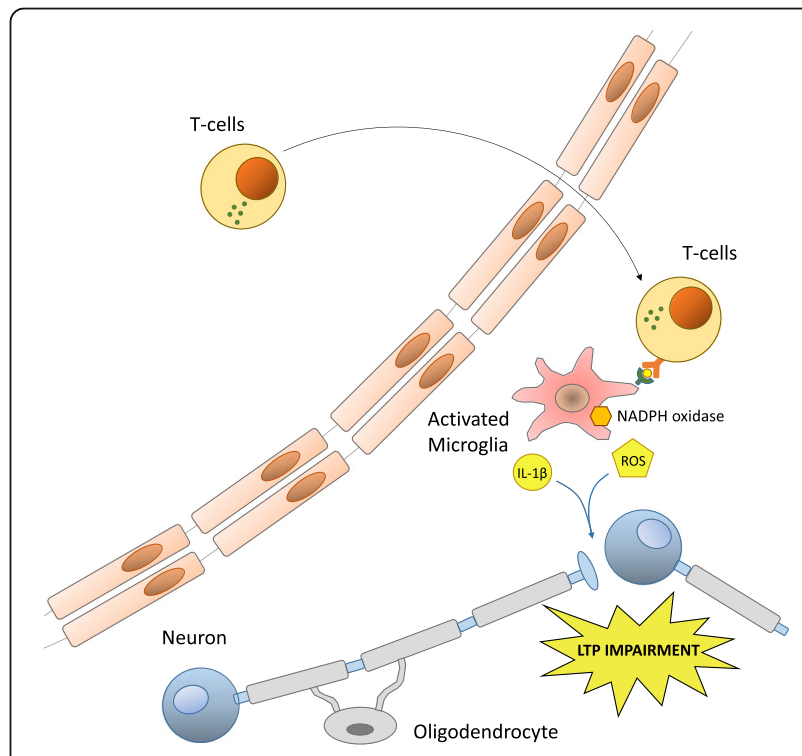

Fig. 3 Neuroinflammation and synaptic impairment. According to a widely accepted pathogenic model for MS and experimental autoimmune encephalomyelitis (EAE), autoreactive B and T cells migrate in the CNS through the blood-brain barrier. At this site, microglial cells participate in the process of $T$ cells reactivation presenting CNS antigens in association with MHC class ॥ molecules. Immune cells trigger the neuroinflammatory process associated with MS and EAE, with the production of cytokines (such as $\mathrm{IL}-1 \beta$ and TNF-a) and other soluble products of inflammation (such as ROS) capable to deeply influence neuronal transmission, potentially leading to the disruption of the main form of synaptic plasticity (LTP). In this process, microglial activation is associated with an overexpression of NADPH-oxidase, a ROS producing enzyme, which has been demonstrated to be relevant for synaptic plasticity impairment during neuro-inflammatory processes [58] 


\section{Hippocampal involvement during MS}

Different histological studies demonstrated that hippocampus is extensively involved during MS, since hippocampal demyelination has been detected in 53\% to $79 \%$ of post-mortem MS brains [15]. Interestingly, Dutta and colleagues showed that hippocampal demyelination is associated with a pronounced decrease in synaptic density, with minimal neuronal loss, and an altered neuronal expression of genes involved in synaptic plasticity, axonal transport and memory/learning, such as glutamate receptors [16]. This synaptic pauperization could contribute to cognitive dysfunction [16]. During last years, neuroimaging studies showed that hippocampal atrophy is associated with memory dysfunction and depression $[18,29,89]$. In particular, a recent multicentre study used several structural MRI modalities to assess how the involvement of different brain regions links to the specific cognitive deficit suffered by the patient [89]. The authors found that atrophy of left postcentral gyrus and right hippocampus represents one of the best MRI findings useful to discriminate between patients with cognitive deficits and patients cognitively preserved [89].

Together with structural studies, functional MRI highlighted the key role played by the hippocampus in cognitively impaired patients. Hulst and colleagues investigated functional hippocampal activation and its connectivity during specific memory tasks in cognitively preserved and cognitively impaired MS patients [90]. They found that, in addition to a decreased hippocampal volume, patients with memory impairment show a decreased activation of the right hippocampus and an increased functional connectivity of the left hippocampus, mostly in connection with right posterior cingulate region, during cognitive performance [90]. Hippocampus and posterior cingulate region are both included in brain default mode network (DMN), a medial cortical network which has been demonstrated to be impaired during Alzheimer's disease [91, 92]. Interestingly, an increased connectivity between the hippocampus and other brain structures involved in the DMN was linked to cognitive impairment during MS [93]. Hulst and colleagues hypothesized that the increased functional connectivity could represent a maladaptive response of the memory functional pathway to the accumulating damage occurring during MS, possibly related to the disinhibition of the entire DMN [90]. In fact, white matter lesions disseminated throughout the brain could impair functional connectivity between the hippocampus and other brain regions located in the frontal, temporal, parietal lobe and cingulate cortex, ultimately leading to a disconnection syndrome when the compensatory hippocampal circuitries fail. This was the hypothesis raised by Rocca and colleagues, who showed a strong correlation between a high brain T2 lesion volume, reduced hippocampal resting-state functional connectivity, depressive symptoms and clinical disability in MS patients [17]. A recent study characterized the relationship existing among neuro-inflammation, hippocampal functional connectivity and depressive symptoms during MS, by using positron emission tomography (PET) and functional MRI [94]. The authors measured hippocampal microglia activation in patients with MS using the 18$\mathrm{kDa}$ translocator protein (TSPO) radio ligand [18 $\mathrm{F}]$ PBR111, the Beck Depression Inventory (BDI) to assess depressive symptoms, and resting-state functional MRI to study hippocampal functional connectivity [94]. As results, the authors found that intense microglial activation within the hippocampus was correlated with a reduced hippocampal connectivity and severe depression [94]. Moreover, another research group recently quantified the presence of activated macrophages/microglia with simultaneous MR-PET imaging, using the TSPO radio ligand ${ }^{11} \mathrm{C}$-PBR28, in patients with both relapsingremitting and secondary progressive MS [95]. Relative to controls, MS subjects exhibited abnormally high brain ${ }^{11} \mathrm{C}$-PBR28 binding, the greatest increases being in cortex and cortical lesions, thalamus, hippocampus, and normally appearing white matter (NAWM) [95]. Interestingly, the authors found that microglia/macrophages activation correlated with reduced cognitive performances in the investigated cohort. Specifically, increased TSPO levels in the hippocampus, as well as in the thalamus and NAWM were associated with impaired Symbol Digit Modalities Test (SDMT) performances, a measure of information-processing speed [95].

In conclusion, all these studies showed that the hippocampus could be directly and indirectly affected by inflammation/demyelination with a subsequent alteration of synaptic density and neuronal transmission. Moreover, also demyelinating lesions spreading throughout the brain could indirectly determine a disconnection syndrome, affecting normal hippocampal functional connectivity, both during resting condition and memory tasks. Neuro-inflammation, and in particular microglial activation, could contribute to alter normal hippocampal connectivity with other key brain regions involved in the maintenance of a normal affective state and cognition [94, 95].

\section{Conclusions}

During the last decades, many studies explored the presence of structural and functional hippocampal changes and their characteristics during neuro-inflammatory processes. A reduction of hippocampal volume was found to be associated to cognitive deficits involving information processing speed and visuo-spatial, verbal and episodic memory during MS [96]. It has been suggested that hippocampal impairment could take place early 
during the course of MS, not directly relating to the demyelinating lesions [31]. Indeed, a selective bilateral hippocampal CA1 atrophy, exceeding global brain volume loss, was found in patients with relapsing-remitting MS independently from T2-lesion volumes, in association with deficits in memory encoding, but not in the speed of information processing, as assessed with wordlist learning test and paced auditory serial addition task (PASAT) [31]. In this scenario, functional hippocampal synaptic plasticity impairment could play a fundamental role in cognitive deficits and symptoms progression during MS. Electrophysiological analysis of synaptic transmission in experimental models of MS showed that hippocampal synaptic plasticity is altered during CNS inflammation, probably due to the activation of microglia and the release of soluble inflammatory products, worsening the performances in hippocampal-related behavioral tests [13]. Accordingly, the presence of active inflammation, represented by isolated gadolinium enhancing lesions at brain MRI, was demonstrated to be associated with poorer cognitive performances in MS patients [97]. An altered synaptic plasticity could affect hippocampal functional connectivity, worsening the disconnection syndrome associated with demyelination and synaptic loss within the hippocampus. In conclusion, the hippocampus emerges as a structure particularly vulnerable to injury during neuro-inflammatory processes. It is affected early during the course of the disease, as assessed by several studies on both experimental animal models of MS and patients suffering with MS. A better understanding of the pathogenic process leading to this selective structural and functional hippocampal impairment appears fundamental to imagine new therapeutic approaches, targeted to preserve cognitive functions and quality of life in people with MS.

\footnotetext{
Abbreviations

AMPA: a-amino-3-hydroxy-5-methyl-4-isoxazolepropionic acid; AP5: Aminophosphonovaleric acid; AQP4: Aquaporin-4; BDI: Beck depression inventory; BDNF: Brain derived neurotrophic factor; CA: Cornus ammonis; CaMKII: Calcium/calmodulin-dependent protein kinase II; CFA: Complete freund's adjuvant; CNS: Central nervous system; CNTF: Ciliary neurotrophic factor; CSF: Cerebro-spinal fluid; CX3CR1: Fractalkine; DG: Dentate gyrus; DMN: Default mode network; EAE: Experimental autoimmune encephalomyelitis; EC: Entorhinal cortex; EPSP: Excitatory post-synaptic potential; FGF-2: Fibroblast growth factor 2; GDNF: Glial cell-line derived neurotrophic factor; Glu: Glutamate; GSH: Glutathione; HFS: High frequency stimulation; IAT: Inhibitory avoidance test; IL-1 $\beta$ : Interleukin-1 $\beta$; LTD: Longterm depression; LTP: Long-term potentiation; MAPK: Mitogen-activatedactivated protein kinase; MF: Mossy fibers; MOG $_{35-55}$ : Myelin oligodendrocyte glycoprotein 35-55; MRI: Magnetic resonance imaging; MS: Multiple sclerosis; NADPH: Nicotinamide adenine dinucleotide phosphate; NAWM: Normally appearing white matter; NF-k $\beta$ : Nuclear factor-kappa $\beta$; NGF: Nerve growth factor; NMDA: N-methyl-D-aspartate; NO: Nitric oxide; PASAT: Paced auditory serial addition task; PET: Positron emission tomography; PKA: Protein kinase A; PP: Performant path; ROS: Reactive oxygen species; SC: Schaffer collaterals; SDMT: Symbol digit modalities test; SUB: Subiculum; TMS: Transcranial magnetic stimulation; TNF-a: Tumor necrosis factor a; TSPO: 18-kDa translocator protein
}

\section{Acknowledgements}

This study was supported by Fondazione Italiana Sclerosi Multipla (FISM) (to P.C., project codes 2010/R/10 and 2011/R/10) and by Ministero della Salute Ricerca Finalizzata - Bando Giovani Ricercatori (to M.D.F., project code GR2010-2312924)

\section{Availability of data and materials \\ Not applicable.}

\section{Authors' contributions}

AM and MDF conceived the review. AM, MDF and AT performed literature review on hippocampal synaptic plasticity in EAE. AM, MDG and LG performed literature review on hippocampal involvement in MS. AM wrote the initial draft of the manuscript. AM and VG prepared the figures. MDF and $P C$ reviewed the manuscript draft. All authors read and approved the final manuscript.

\section{Competing interests}

AM received travel grants from Teva and Sanofi Genzyme to attend national conferences. LG received travel grants from Biogen-ldec, Biogen, Novartis, Teva, Genzyme and Almirall to attend national and international conferences. MDF participated to advisory boards of Biogen Idec, Teva, Bayer, and Novartis; received travel grants from Bayer Schering, Biogen Dompé, Biogen Idec, Merck Serono, Novartis and Sanofi Genzyme to attend national and international conferences and speaker and writing honoraria from Biogen Idec, Novartis and Sanofi-Genzyme. MDF received research support from Associazione Italiana Sclerosi Multipla (AISM) and the Italian Minister of Health. MDG received travel grants from Biogen, Novartis, Teva and Merk-Serono to attend national and international conferences. PC received/receives research support from Bayer Schering, Biogen:Dompé, Boehringer Ingelheim, Eisai, Lundbeck, Merck:Serono, Novartis, Sanofi:Aventis, Sigma:Tau and UCB Pharma. PC also receives/received support from Ricerca Corrente IRCCS, Ricerca Finalizzata IRCCS, European Community Grant REPLACES (restorative plasticity at corticostriatal excitatory synapses), the Italian Minister of Health, and AIFA (Agenzia Italiana del Farmaco). AT and VG report no competing interests.

\section{Consent for publication}

Not applicable.

\section{Ethics approval and consent to participate}

Not applicable, review article.

\section{Author details}

${ }^{1}$ Clinica Neurologica, Dipartimento di Medicina, Università degli Studi di Perugia, Ospedale Santa Maria della Misericordia, S. Andrea delle Fratte, Perugia 06132, Italy. ${ }^{2}$ IRCCS, Fondazione Santa Lucia, via del Fosso di Fiorano 64, Rome 00143, Italy. ${ }^{3}$ Sezione di Fisiologia e Biochimica, Dipartimento di Medicina Sperimentale, Università degli Studi di Perugia, S. Andrea delle Fratte, Perugia 06132, Italy. ${ }^{4}$ Dipartimento di Filosofia, Scienze Sociali, Umane, e della Formazione, Università degli Studi di Perugia, Perugia, Italy.

Received: 26 September 2016 Accepted: 4 January 2017 Published online: 26 January 2017

\section{References}

1. Di Filippo M, Sarchielli P, Picconi B, Calabresi P. Neuroinflammation and synaptic plasticity: theoretical basis for a novel, immune-centred, therapeutic approach to neurological disorders. Trends Pharmacol Sci. 2008;29:402-12.

2. Salter MW, Beggs S. Sublime microglia: expanding roles for the guardians of the CNS. Cell. 2014;158(1):15-24.

3. Morris GP, Clark IA, Zinn R, Vissel B. Microglia: a new frontier for synaptic plasticity, learning and memory, and neurodegenerative disease research. Neurobiol Learn Mem. 2013;105:40-53.

4. Wake H, Moorhouse AJ, Miyamoto A, Nabekura J. Microglia: actively surveying and shaping neuronal circuit structure and function. Trends Neurosci. 2013;36(4):209-17.

5. Bains JS, Oliet SH. Glia: they make your memories stick! Trends Neurosci. 2007;30(8):417-24. 
6. Cibelli M, Fidalo AR, Terrando N, Ma D, Monaco C, Feldmann M, Tarata M, Lever IJ, Nanchahal J, Fanselow MS, Maze M. Role of interleukin-1beta in postoperative cognitive dysfunction. Ann Neurol. 2010;68:360-8.

7. Dantzer R, O'Connor JC, Freund GG, Johnson RW, Kelley KW. From inflammation to sickness and depression: when the immune system subjugates the brain. Nat Rev Neurosci. 2008;9:46-56.

8. Konsman JP, Parnet P, Dantzer R. Cytokine-induced sickness behaviour: mechanisms and implications. Trends Neurosci. 2002;25:154-9.

9. Chiaravalloti ND, DeLuca J. Cognitive impairment in multiple sclerosis. Lancet Neurol. 2008;7:1139-51.

10. Rocca MA, Amato MP, De Stefano N, Enzinger C, Geurts JJ, Penner IK, Rovira A, Sumowski JF, Valsasina P, Filippi M. MAGNIMS Study Group. Clinical and imaging assessment of cognitive dysfunction in multiple sclerosis. Lancet Neurol. 2015;14(3):302-17. 2015 Feb 4.

11. Centonze D, Muzio L, Rossi S, Cavasinni F, De Chiara V, Bergami A, Musella A, D'Amelio M, Cavallucci V, Martorana A, Bergamaschi A, Cencioni MT, Diamantini A, Butti E, Comi G, Bernardi G, Cecconi F, Battistini L, Furlan R, Martino G. Inflammation triggers synaptic alteration and degeneration in experimental autoimmune encephalomyelitis. J Neurosci. 2009;29(11):3442-52.

12. Mandolesi G, Gentile A, Musella A, Fresegna D, De Vito F, Bullitta S, Sepman H, Marfia GA, Centonze D. Synaptopathy connects inflammation and neurodegeneration in multiple sclerosis. Nat Rev Neurol. 2015;11(12):711-24.

13. Di Filippo M, de lure A, Durante V, Gaetani L, Mancini A, Sarchielli P, Calabresi P. Synaptic plasticity and experimental autoimmune encephalomyelitis: implications for multiple sclerosis. Brain Res. 2015;1621:205-13.

14. Pelletier J, Audoin B, Reuter F, Ranjeva J. Plasticity in MS: from functional imaging to rehabilitation. Int MS J. 2009;16(1):26-31.

15. Geurts JJ, Bö L, Roosendaal SD, Hazes T, Daniëls R, Barkhof F, Witter MP, Huitinga I, van der Valk P. Extensive hippocampal demyelination in multiple sclerosis. J Neuropathol Exp Neurol. 2007:66(9):819-27.

16. Dutta R, Chang A, Doud MK, Kidd GJ, Ribaudo MV, Young EA, Fox RJ, Staugaitis SM, Trapp BD. Demyelination causes synaptic alterations in hippocampi from multiple sclerosis patients. Ann Neurol. 2011;69(3):445-54.

17. Rocca MA, Pravatà $E$, Valsasina $P$, Radaelli M, Colombo B, Vacchi L, Gobbi C, Comi G, Falini A, Filippi M. Hippocampal-DMN disconnectivity in MS is related to WM lesions and depression. Hum Brain Mapp. 2015; 36(12):5051-63.

18. Gold SM, O'Connor MF, Gill R, Kern KC, Shi Y, Henry RG, Pelletier D, Mohr DC, Sicotte NL. Detection of altered hippocampal morphology in multiple sclerosis-associated depression using automated surface mesh modeling. Hum Brain Mapp. 2014;35(1):30-7.

19. Roosendaal SD, Moraal B, Vrenken H, Castelijns JA, Pouwels PJ, Barkhof F Geurts JJ. In vivo MR imaging of hippocampal lesions in multiple sclerosis. J Magn Reson Imaging. 2008;27(4):726-31.

20. Longoni G, Rocca MA, Pagani E, Riccitelli GC, Colombo B, Rodegher M, Falini A, Comi G, Filippi M. Deficits in memory and visuospatial learning correlate with regional hippocampal atrophy in MS. Brain Struct Funct. 2015;220(1): 435-44

21. Scoville WB, Milner B. Loss of recent memory after bilateral hippocampa lesions. J Neurol Neurosurg Psychiatry. 1957;20(1):11-21.

22. Burgess N, Maguire EA, O'Keefe J. The human hippocampus and spatial and episodic memory. Neuron. 2002;35(4):625-41.

23. Eichenbaum $\mathrm{H}$. Hippocampus: cognitive processes and neural representations that underlie declarative memory. Neuron. 2004;44(1):109-20.

24. Di Filippo M, Calabresi P. Ischemic bilateral hippocampal dysfunction during transient global amnesia. Neurology. 2007;69(5):493.

25. O'Keefe J, Nadel L. The hippocampus as a cognitive map. Oxford: Oxford University Press; 1978.

26. Zucker HR, Ranganath C. Navigating the human hippocampus without a GPS. Hippocampus. 2015;25(6):697-703.

27. Bannerman DM, Sprengel R, Sanderson DJ, McHugh SB, Rawlins JN, Monyer H, Seeburg PH. Hippocampal synaptic plasticity, spatial memory and anxiety. Nat Rev Neurosci. 2014;15(3):181-92.

28. Lee DW, Miyasato LE, Clayton NS. Neurobiological bases of spatial learning in the natural environment: neurogenesis and growth in the avian and mammalian hippocampus. Neuroreport. 1998;9(7):R15-27.

29. Sherry DF, Jacobs LF, Gaulin SJ. Spatial memory and adaptive specialization of the hippocampus. Trends Neurosci. 1992;15(8):298-303.

30. Maguire EA, Gadian DG, Johnsrude IS, Good CD, Ashburner J, Frackowiak RS, Frith CD. Navigation-related structural change in the hippocampi of taxi drivers. Proc Natl Acad Sci U S A. 2000;97(8):4398-403.
31. Sicotte NL, Kern KC, Giesser BS, Arshanapalli A, Schultz A, Montag M, Wang H, Bookheimer SY. Regional hippocampal atrophy in multiple sclerosis. Brain. 2008;131(Pt 4):1134-41.

32. Pastalkova E, Serrano P, Pinkhasova D, Wallace E, Fenton AA, Sacktor TC Storage of spatial information by the maintenance mechanism of LTP. Science. 2006:313(5790):1141-4.

33. Tsien JZ, Huerta PT, Tonegawa S. The essential role of hippocampal CA NMDA receptor-dependent synaptic plasticity in spatial memory. Cell. 1996;87(7):1327-38

34. Neves G, Cooke SF, Bliss TV. Synaptic plasticity, memory and the hippocampus: a neural network approach to causality. Nat Rev Neurosci. 2008;9(1):65-75

35. Bliss TV, Lomo T. Long-lasting potentiation of synaptic transmission in the dentate area of the anaesthetized rabbit following stimulation of the perforant path. J Physiol. 1973;232(2):331-56.

36. Malenka RC, Nicoll RA. Long-term potentiation, a decade of progress? Science. 1999:285(5435):1870-4

37. Hebb DO. The organization of behavior. New York: Wiley; 1949.

38. Morris RG. D.O. Hebb: The Organization of Behavior, Wiley: New York; 1949. Brain Res Bull. 1999 Nov-Dec;50(5-6):437.

39. Bliss TV, Collingridge GL. A synaptic model of memory: long-term potentiation in the hippocampus. Nature. 1993;361:31-9.

40. Malenka RC, Bear MF. LTP and LTD: an embarrassment of riches. Neuron. 2004;44(1):5-21.

41. Dudek SM, Bear MF. Homosynaptic long-term depression in area CA1 of hippocampus and effects of $\mathrm{N}$-methyl-D-aspartate receptor blockade. Proc Natl Acad Sci U S A. 1992;89(10):4363-7.

42. Whitlock JR, Heynen AJ, Shuler MG, Bear MF. Learning induces long-term potentiation in the hippocampus. Science. 2006;313(5790):1093-7.

43. Collingridge GL, Kehl SJ, McLennan H. The antagonism of amino acidinduced excitations of rat hippocampal CA1 neurones in vitro. J Physiol. 1983:334:19-31.

44. Morris RG, Anderson E, Lynch GS, Baudry M. Selective impairment of learning and blockade of long-term potentiation by an N-methyl-Daspartate receptor antagonist, AP5. Nature. 1986;319(6056):774-6.

45. Panatier A, Robitaille R. Astrocytic mGluR5 and the tripartite synapse. Neuroscience. 2016;323:29-34.

46. Clark AK, Gruber-Schoffnegger D, Drdla-Schutting R, Gerhold KJ, Malcangio M, Sandkühler J. Selective activation of microglia facilitates synaptic strength. J Neurosci. 2015:35(11):4552-70.

47. Schafer DP, Lehrman EK, Stevens B. The "quad-partite" synapse: microgliasynapse interactions in the developing and mature CNS. Glia. 2013;61(1):24-36.

48. Miyamoto A, Wake H, Moorhouse AJ, Nabekura J. Microglia and synapse interactions: fine tuning neural circuits and candidate molecules. Front Cell Neurosci. 2013:7:70.

49. Scharfman HE, Binder DK. Aquaporin-4 water channels and synaptic plasticity in the hippocampus. Neurochem Int. 2013;63(7):702-11.

50. Szu Jl, Binder DK. The role of astrocytic aquaporin-4 in synaptic plasticity and learning and memory. Front Integr Neurosci. 2016;10:8.

51. Hamilton NB, Attwell D. Do astrocytes really exocytose neurotransmitters? Nat Rev Neurosci. 2010;11(4):227-38.

52. Volterra A, Meldolesi J. Astrocytes, from brain glue to communication elements: the revolution continues. Nat Rev Neurosci. 2005:6(8):626-40.

53. Ota Y, Zanetti AT, Hallock RM. The role of astrocytes in the regulation of synaptic plasticity and memory formation. Neural Plast. 2013;2013:185463.

54. Bélanger M, Magistretti PJ. The role of astroglia in neuroprotection. Dialogues Clin Neurosci. 2009:11(3):281-95.

55. Mayo L, Trauger SA, Blain M, Nadeau M, Patel B, Alvarez Jl, Mascanfroni ID, Yeste A, Kivisa P, Kallas K, Ellezam B, Bakshi R, Prat A, Antel JP, Weiner HL, Quintana FJ. Regulation of astrocyte activation by glycolipids drives chronic CNS inflammation. Nat Med. 2014;20(10):1147-56.

56. Rostami A, Ciric B. Astrocyte-derived lactosylceramide implicated in multiple sclerosis. Nat Med. 2014;20(10):1092-3.

57. Laurier-Laurin ME, De Montigny A, Attiori Essis S, Cyr M, Massicotte G. Blockade of lysosomal acid ceramidase induces GluN2B-dependent Tau phosphorylation in rat hippocampal slices. Neural Plast. 2014;2014:196812.

58. Di Filippo M, de lure A, Giampà C, Chiasserini D, Tozzi A, Orvietani PL, Ghiglieri V, Tantucci M, Durante V, Quiroga-Varela A, Mancini A, Costa C Sarchielli P, Fusco FR, Calabresi P. Persistent activation of microglia and NADPH oxidase drive hippocampal dysfunction in experimental multiple sclerosis. Sci Rep. 2016;6:20926. 
59. Aguzzi A, Barres BA, Bennett ML. Microglia: scapegoat, saboteur, or something else? Science. 2013;339(6116):156-61.

60. Davalos D, Grutzendler J, Yang G, Kim JV, Zuo Y, Jung S, Littman DR, Dustin ML, Gan WB. ATP mediates rapid microglial response to local brain injury in vivo. Nat Neurosci. 2005;8(6):752-8.

61. Nimmerjahn A, Kirchhoff F, Helmchen F. Resting microglial cells are highly dynamic surveillants of brain parenchyma in vivo. Science. 2005;308(5726): 1314-8.

62. Kaindl AM, Koppelstaetter A, Nebrich G, Stuwe J, Sifringer M, Zabel C, Klose J, Ikonomidou C. Brief alteration of NMDA or GABAA receptor-mediated neurotransmission has long term effects on the developing cerebral cortex. Mol Cell Proteomics. 2008;7(12):2293-310.

63. Paolicelli RC, Bolasco G, Pagani F, Maggi L, Scianni M, Panzanelli P, Giustetto M, Ferreira TA, Guiducci E, Dumas L, Ragozzino D, Gross CT. Synaptic pruning by microglia is necessary for normal brain development. Science. 2011; 333(6048):1456-8.

64. Parkhurst CN, Yang G, Ninan I, Savas JN, Yates 3rd JR, Lafaille JJ, Hempstead BL, Littman DR, Gan WB. Microglia promote learningdependent synapse formation through brain-derived neurotrophic factor. Cell. 2013;155(7):1596-609.

65. Goshen I, Kreisel T, Ounallah-Saad H, Renbaum P, Zalzstein Y, Ben-Hur T, Levy-Lahad E, Yirmiya R. A dual role for interleukin-1 in hippocampaldependent memory processes. Psychoneuroendocrinology. 2007;32(8-10): 1106-15.

66. Vereker $E, O^{\prime}$ Donnell $E$, Lynch MA. The inhibitory effect of interleukin-1beta on long-term potentiation is coupled with increased activity of stressactivated protein kinases. J Neurosci. 2000;20(18):6811-9.

67. Katsuki H, Nakai S, Hirai Y, Akaji K, Kiso Y, Satoh M. Interleukin-1 beta inhibits long-term potentiation in the CA3 region of mouse hippocampal slices. Eur J Pharmacol. 1990;181:323-6.

68. Di Filippo M, Chiasserini D, Gardoni F, Viviani B, Tozzi A, Giampà C, Costa C, Tantucci M, Zianni E, Boraso M, Siliquini S, de lure A, Ghiglieri V, Colcelli E, Baker D, Sarchielli P, Fusco FR, Di Luca M, Calabresi P. Effects of central and peripheral inflammation on hippocampal synaptic plasticity. Neurobiol Dis. 2013;52:229-36

69. Bellinger FP, Madamba S, Siggins GR. Interleukin 1 beta inhibits synaptic strength and long-term potentiation in the rat CA1 hippocampus. Brain Res. 1993;628:227-34.

70. Murray CA, Lynch MA. Evidence that increased hippocampal expression of the cytokine interleukin-1 beta is a common trigger for age- and stressinduced impairments in longterm potentiation. J Neurosci. 1998;18:2974-81.

71. Schneider H, Pitossi F, Balschun D, Wagner A, del Rey A, Besedovsky HO. A neuromodulatory role of interleukin-1 beta in the hippocampus. Proc Natl Acad Sci U S A. 1998;95:7778-83.

72. Avital A, Goshen I, Kamsler A, Segal M, Iverfeldt K, Richter-Levin G, Yirmiya R. Impaired interleukin-1 signaling is associated with deficits in hippocampal memory processes and neural plasticity. Hippocampus. 2003;13:826-34.

73. Rossi S, Studer V, Motta C, Germani G, Macchiarulo G, Buttari F, Mancino R, Castelli M, De Chiara V, Weiss S, Martino G, Furlan R, Centonze D. Cerebrospinal fluid detection of interleukin-1 $\beta$ in phase of remission predicts disease progression in multiple sclerosis. J Neuroinflammation. 2014;11:32.

74. Jones RS, Lynch MA. How dependent is synaptic plasticity on microglial phenotype? Neuropharmacology. 2015;96(Pt A):3-10.

75. Fletcher JM, Lalor SJ, Sweeney CM, Tubridy N, Mills KH. T cells in multiple sclerosis and experimental autoimmune encephalomyelitis. Clin Exp Immunol. 2010;162:1-11.

76. Amor S, Smith PA, Hart B, Baker D. Biozzi mice: of mice and human neurological diseases. J Neuroimmunol. 2005;165:1-10.

77. Baker D, Jackson SJ. Models of multiple sclerosis. ACNR. 2007;6:10-2

78. Kim do Y, Hao J, Liu R, Turner G, Shi FD, Rho JM. Inflammation-mediated memory dysfunction and effects of a ketogenic diet in a murine model of multiple sclerosis. PLoS One. 2012;7(5):e35476

79. Dutra RC, Moreira EL, Alberti TB, Marcon R, Prediger RD, Calixto JB. Spatial reference memory deficits precede motor dysfunction in an experimental autoimmune encephalomyelitis model: the role of kallikrein-kinin system. Brain Behav Immun. 2013;33:90-101.

80. Mosayebi G, Soleyman MR, Khalili M, Mosleh M, Palizvan MR. Changes in synaptic transmission and long-term potentiation induction as a possible mechanism for learning disability in an animal model of multiple sclerosis. Int Neurourol J. 2016;20(1):26-32.
81. Gardoni F, Mauceri D, Malinverno M, Polli F, Costa C, Tozzi A, Siliquini S, Picconi B, Cattabeni F, Calabresi P, Di Luca M. Decreased NR2B subunit synaptic levels cause impaired long-term potentiation but not long-term depression. J Neurosci. 2009;29:669-77.

82. Foster KA, McLaughlin N, Edbauer D, Phillips M, Bolton A, Constantine-Paton M, Sheng M. Distinct roles of NR2A and NR2B cytoplasmic tails in long-term potentiation. J Neurosci. 2010;30:2676-85.

83. Novkovic T, Shchyglo O, Gold R, Manahan-Vaughan D. Hippocampal function is compromised in an animal model of multiple sclerosis. Neuroscience. 2015;309:100-12.

84. Nisticò R, Mango D, Mandolesi G, Piccinin S, Berretta N, Pignatelli M, Feligioni M, Musella A, Gentile A, Mori F, Bernardi G, Nicoletti F, Mercuri NB, Centonze D. Inflammation subverts hippocampal synaptic plasticity in experimental multiple sclerosis. PLoS One. 2013;8(1):e54666.

85. Prochnow N, Gold R, Haghikia A. An electrophysiologic approach to quantify impaired synaptic transmission and plasticity in experimental autoimmune encephalomyelitis. J Neuroimmunol. 2013;264(1-2):48-53.

86. Weiss S, Mori F, Rossi S, Centonze D. Disability in multiple sclerosis: when synaptic long-term potentiation fails. Neurosci Biobehav Rev. 2014:43:88-99.

87. Mori F, Kusayanagi H, Nicoletti CG, Weiss S, Marciani MG, Centonze D. Cortical plasticity predicts recovery from relapse in multiple sclerosis. Mult Scler. 2014:20(4):451-7.

88. Mori F, Rossi S, Piccinin S, Motta C, Mango D, Kusayanagi H, Bergami A, Studer V, Nicoletti CG, Buttari F, Barbieri F, Mercuri NB, Martino G, Furlan R, Nisticò R, Centonze D. Synaptic plasticity and PDGF signaling defects underlie clinical progression in multiple sclerosis. J Neurosci. 2013;33(49): 19112-9.

89. Preziosa P, Rocca MA, Pagani E, Stromillo ML, Enzinger C, Gallo A, Hulst HE, Atzori M, Pareto D, Riccitelli GC, Copetti M, De Stefano N, Fazekas F, Bisecco A, Barkhof F, Yousry TA, Arévalo MJ, Filippi M. MAGNIMS study group. Structural MRI correlates of cognitive impairment in patients with multiple sclerosis: a multicenter study. Hum Brain Mapp. 2016;37(4):1627-44.

90. Hulst HE, Schoonheim MM, Van Geest Q, Uitdehaag BM, Barkhof F, Geurts JJ. Memory impairment in multiple sclerosis: relevance of hippocampal activation and hippocampal connectivity. Mult Scler. 2015;21(13):1705-12.

91. Greicius MD, Srivastava G, Reiss AL, Menon V. Default mode network activity distinguishes Alzheimer's disease from healthy aging: Evidence from functional MRI. Proc Natl Acad Sci U S A. 2004;101:4637-42.

92. Kenny ER, Blamire AM, Firbank MJ, O'Brien JT. Functional connectivity in cortical regions in dementia with Lewy bodies and Alzheimer's disease. Brain. 2012;135:569-81.

93. Hawellek DJ, Hipp JF, Lewis CM, Corbetta M, Engel AK. Increased functional connectivity indicates the severity of cognitive impairment in multiple sclerosis. Proc Natl Acad Sci U S A. 2011;108:19066-71.

94. Colasanti A, Guo Q, Giannetti P, Wall MB, Newbould RD, Bishop C, Onega M, Nicholas R, Ciccarelli O, Muraro PA, Malik O, Owen DR, Young AH, Gunn RN, Piccini P, Matthews PM, Rabiner EA. Hippocampal neuroinflammation, functional connectivity, and depressive symptoms in multiple sclerosis. Biol Psychiatry. 2015. S0006-3223(15)01025-2.

95. Herranz E, Giannì C, Louapre C, Treaba CA, Govindarajan ST, Ouellette R, Loggia ML, Sloane JA, Madigan N, Izquierdo-Garcia D, Ward N, Mangeat G, Granberg T, Klawiter EC, Catana C, Hooker JM, Taylor N, lonete C, Kinkel RP, Mainero C. Neuroinflammatory component of gray matter pathology in multiple sclerosis. Ann Neurol. 2016;80(5):776-90.

96. Koenig KA, Sakaie KE, Lowe MJ, Lin J, Stone L, Bermel RA, Beall EB, Rao SM, Trapp BD, Phillips MD. Hippocampal volume is related to cognitive decline and fornicial diffusion measures in multiple sclerosis. Magn Reson Imaging. 2014;32(4):354-8.

97. Bellmann-Strobl J, Wuerfel J, Aktas O, Dörr J, Wernecke KD, Zipp F, Paul F. Poor PASAT performance correlates with MRI contrast enhancement in multiple sclerosis. Neurology. 2009;73(20):1624-7. 